\title{
Resveratrol suppresses human hepatocellular carcinoma via targeting HGF-c-Met signaling pathway
}

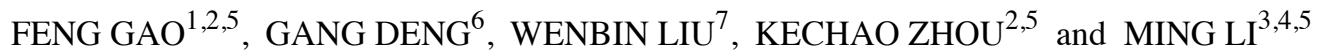 \\ ${ }^{1}$ Department of Ultrasonography, The Third Xiangya Hospital of Central South University, Changsha, \\ Hunan 410013; ${ }^{2}$ Powder Metallurgy Research Institute of Central South University, Changsha, Hunan 410083; \\ ${ }^{3}$ Changsha Stomatological Hospital, Changsha, Hunan 410004; ${ }^{4}$ School of Stomatology, Hunan University of \\ Traditional Chinese Medicine, Changsha, Hunan 410208; ${ }^{5}$ Postdoctoral Station of Materials Science and Engineering \\ of Central South University, Changsha, Hunan 410083; ${ }^{6}$ Department of Hepatobiliary and Pancreatic Surgery, \\ The Third Xiangya Hospital of Central South University, Changsha; ${ }^{7}$ Hunan Cancer Hospital and The Affiliated \\ Cancer Hospital of Xiangya School of Medicine, Central South University, Changsha, Hunan 410013, P.R. China
}

Received July 31, 2016; Accepted December 27, 2016

DOI: 10.3892/or.2017.5347

\begin{abstract}
Resveratrol, one of the major polyphenols found in red wine, is suggested to have a role as a chemoprevention or chemotherapy agent in various human cancer models. Herein, we report that resveratrol has a profound antitumor effect on human hepatocellular carcinoma (HCC) cells by downregulation of the HGF-c-Met signaling pathway. Resveratrol inhibited anchorage-dependent and -independent growth of HCC cells in a dose-dependent manner. Short-term resveratrol exposure substantially decreased HGF-induced c-Met signaling pathway activation, and long-term exposure to resveratrol markedly inhibited c-Met expression on the cell membrane. Additionally, resveratrol suppressed HGF-induced cell invasion, and knockdown of c-Met decreased the sensitivity of HCC cells to resveratrol treatment. Finally, the antitumor activity of resveratrol was validated in xenograft model and resveratrol prominently restrained tumor growth in vivo. In summary, our results suggested that c-Met offers a candidate molecular target for hepatocellular carcinoma management.
\end{abstract}

Correspondence to: Dr Kechao Zhou, Powder Metallurgy Research Institute of Central South University, Changsha, Hunan 410083, P.R. China

E-mail: kechao_zhouCSU@163.com

Dr Ming Li, Changsha Stomatological Hospital, Changsha, Hunan 410004, P.R. China

E-mail: mingli_science@163.com

Abbreviations: HCC, hepatocellular carcinoma; HGF, hepatocyte growth factor

Key words: hepatocellular carcinoma, resveratrol, c-Met

\section{Introduction}

Hepatocellular carcinoma (HCC) is one of the most common causes of cancer-related death worldwide. Despite the development of new therapeutic strategies and improved patient care, the 5-year survival rate of $\mathrm{HCC}$ is still dismal at approximately $15 \%$ (1-3). Although the available therapies, including surgical resection and transplantation, have significantly improved survival in patients with early stage tumors, the prognosis of $\mathrm{HCC}$ for advanced-stage disease remains very poor (4). HCC is known to be associated with hepatitis B virus (HBV), hepatitis $\mathrm{C}$ virus, and alcohol abuse. However, other risk factors that are genetic and physiological also contribute to the etiology of HCC $(5,6)$. Therefore, understanding the molecular mechanisms of the complex multistep process of HCC, and identify novel chemical entity with activity against HCC, could facilitate the development of preventive measures, early diagnostic methods, and better treatments.

c-Met, originally identified as a TRP-Met fusion gene from a human osteosarcoma cell line, encodes a prototype member of a distinct subfamily of heterodimeric receptor tyrosine kinases (7). Hepatocyte growth factor (HGF) is the only known high affinity ligand for the c-Met receptor. Binding of HGF to c-Met causes receptor multimerization, phosphorylation, and catalytic activation. The activation of c-Met leads to phosphorylation of multiple downstream effectors, including RAS/MAPK, PI3K/AKT, FAK, and c-SRC, that are essential for regulating cell growth, survival, motility, and metastasis $(8,9)$. Importantly, the overexpression or activating mutations of c-Met has been frequently found in many human solid tumors, such as liver (10), lung (11), colon (12), ovarian (13), breast (14) and gastric (15) cancers. Recently, Zhao et al demonstrated that downregulation of the activation of c-Met via a specific inhibitor, cabozantinib, leading to suppressed epithelial-mesenchymal transition and tumor growth in breast tumors (16). Clinical retrospective analysis revealed that higher expression of c-Met in tumor tissue had positive relationship with patients' poor prognosis (17). 
Resveratrol, a natural product found in red grapes and wine, is one of the most common dietary flavonoids. Except antioxidant and anti-inflammatory effect as reported, recent studies have demonstrated that resveratrol exerted antitumor effects on a panel of human cancers, such as breast (18), colon (19), ovarian (20), lung (21) and prostate cancer (22). Mechanism investigation manifested that multiple signaling pathways and mechanisms are involved in the antitumor activity of resveratrol, including suppression of various protein kinases, such as Akt, ERK and EGFR, induction of cell cycle arrest or apoptosis, and inhibition of cell migration and metastasis (23). Nonetheless, the antitumor activity of resveratrol in HCC, as well as the effect on c-Met signaling pathway, has not yet been fully investigated.

In this study, we first reported that the c-Met signaling pathway was involved in resveratrol-induced tumor suppression in hepatocellular carcinoma cells. Our results suggest that targeting the activation of HGF-c-Met signaling pathway might be an effective preventive and therapeutic pursuit in this kind of tumor.

\section{Materials and methods}

Cell culture and reagents. The HCC cell line Hep3B was purchased from American Type Culture Collection (ATCC). The MHCC97-L and MHCC97-H cells were purchased from Cell Bank of Chinese Academy of Sciences, Shanghai, China. Cells were cytogenetically tested and authenticated before being frozen. Each vial of frozen cells was thawed and maintained for 2 months (10 passages). Of note, Hep3B, MHCC97-L and MHCC97-H were maintained with Dulbecco's modified Eagle's medium (DMEM) containing 10\% FBS and 1\% antibiotics. All cell lines were incubated at $37^{\circ} \mathrm{C}$ in a humidified atmosphere containing $5 \% \mathrm{CO}_{2}$. Resveratrol and chemical reagents, including Tris, $\mathrm{NaCl}$, SDS and DMSO, for molecular biology and buffer preparation were purchased from SigmaAldrich (St. Louis, MO, USA).

MTS assay. The MTS assay was performed as previously described (24). Briefly, human HCC cells were seeded ( $3 \times 10^{3} /$ well $/ 100 \mu 1$ ) into 96 -well plates, and treated with various doses of resveratrol for different time points as indicated. The proliferation was assessed by MTS assay (Promega, Madison, WI, USA) according to instructions provided.

Anchorage-independent cell growth assay. The anchorageindependent cell growth was performed as previously described (25). Briefly, cells were suspended $(8,000$ cells $/ \mathrm{ml})$ in $1 \mathrm{ml}$ of $0.3 \%$ agar with Eagle's basal medium containing $10 \%$ FBS, $1 \%$ antibiotics, and different concentrations of resveratrol overlaid into six-well plates containing a $0.6 \%$ agar base. The cultures were maintained in a $37^{\circ} \mathrm{C}, 5 \% \mathrm{CO}_{2}$ incubator for 2 weeks, and then colonies were counted under a microscope using the Image-Pro Plus software program (Media Cybernetics, Silver Spring, MD, USA).

Invasion assay. An invasion assay was conducted using a modified Boyden chamber and Matrigel-coated (BD Bioscience, San Jose, CA, USA) polycarbonate nucleopore membranes (Corning, Inc., Corning, NY, USA; $8-\mu \mathrm{m}$ pore size). Serum-free medium containing HGF $(10 \mathrm{ng} / \mathrm{ml})$ was pipetted into the lower wells. MHCC $97-\mathrm{H}$ cells were trypsinized and suspended at a density of $1 \times 10^{5}$ cells $/ 100 \mu 1$ in serum-free medium without HGF. Cells were then pretreated with resveratrol for $30 \mathrm{~min}$ and $100 \mu \mathrm{l}$ of the cell suspension were loaded into the upper wells. The chamber was incubated in a $5 \% \mathrm{CO}_{2}$ incubator at $37^{\circ} \mathrm{C}$. After $24 \mathrm{~h}$ of incubation, the membrane was fixed and stained with crystal violet solution. Invasiveness was determined by counting the cells that passed through the filter.

Subcellular proteome fractionation. The subcellular proteome fractions were prepared using the ProteoExtract Subcellular Proteome Extraction kit (539790, Millipore, Temecula, CA, USA) according to the manufacturer's instructions.

Protein preparation and western blotting. Protein samples were extracted with RIPA buffer (10 mM Tris- $\mathrm{Cl}(\mathrm{pH} 8.0)$, $1 \mathrm{mM}$ EDTA, $0.5 \mathrm{mM}$ EGTA, $1 \%$ Triton X-100, 0.1\% sodium deoxycholate, $0.1 \%$ SDS, $140 \mathrm{mM} \mathrm{NaCl}$ ). Protein concentration was determined using the BCA Assay Reagent (Pierce, Rockford, IL, USA). For immunoblotting, proteins $(40 \mu \mathrm{g})$ were detected with specific antibodies and an HRP-conjugated secondary antibody. Primary antibodies were used for immunoblotting: p-c-Met, c-Met, p-Akt, p-ERK1/2 and N-cadherin from Cell Signaling Technology; $\beta$-actin from Sigma-Aldrich, Ki67 from Abcam. Secondary antibodies anti-rabbit IgG HRP and anti-mouse IgG HRP were purchased from Cell Signaling Technology. Antibody conjugates were visualized by chemiluminescence (ECL, Thermo Scientific, Rockford, IL, USA).

Lentiviral infection. To generate knockdown c-Met cells, pLKO.1-sh-GFP or pLKO.1-sh-c-Met lentivirus plasmids, were co-transfected into $293 \mathrm{~T}$ cells with PSPAX2 and $P M D 2-G$. Viral supernatant fractions were collected at $48 \mathrm{~h}$ after transfection and filtered through a $0.45 \mu \mathrm{m}$ filter followed by infection into cells together with $10 \mu \mathrm{g} / \mathrm{ml}$ polybrene. The next day, the medium was replaced with fresh medium containing $1 \mu \mathrm{g} / \mathrm{ml}$ puromycin and cells were incubated for another 6 days.

In vivo tumor growth assay. All the experimentations for animals were approved by the Ethics Committee of Central South University, Changsha, Hunan, China. MHCC97-H cells $\left(2 \times 10^{6}\right)$ in $100 \mu 1$ DMEM medium were inoculated s.c. into the right flank of 6-week-old female athymic nude mice. Nude mice $(n=5)$ were randomly divided to groups when tumor volume reached $50-100 \mathrm{~mm}^{3}$. The dosage of resveratrol was $30 \mathrm{mg} / \mathrm{kg}$ and was administered every three days by intraperitoneal injection, whereas control mice were administered vehicle. The body weight of each mouse was recorded and tumor volume was determined by vernier caliper twice a week. Volume was calculated following the formula of $\mathrm{A} \times \mathrm{B}^{2} \times 0.5$, wherein $\mathrm{A}$ is the longest diameter of tumor, $\mathrm{B}$ is the shortest diameter and $\mathrm{B}^{2}$ is $\mathrm{B}$ squared. Mice were monitored until day 34 and at that time mice were euthanized and tumors extracted.

Immunohistochemical analysis. A Vectastain Elite ABC kit (Vector Laboratories; Burlingame, CA, USA) was used for immunohistochemical staining according to the recommended 

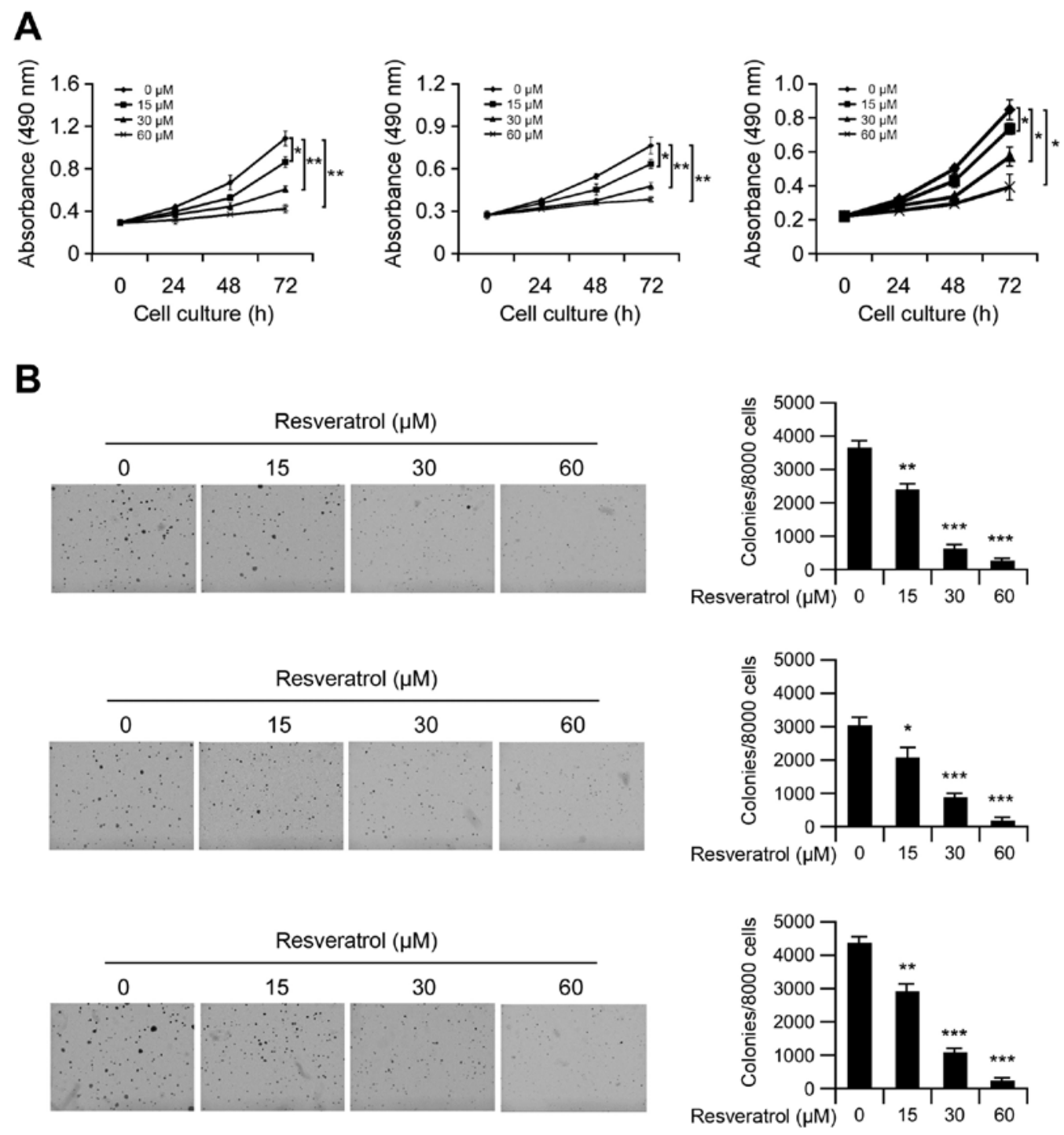

Figure 1. Resveratrol inhibits HCC cell growth in vitro. (A) Resveratrol decreases cell viability. Hep3B (left), MHCC97-L (middle) and MHCC97-H (right) cells were treated or not treated with resveratrol as indicated. (B) Resveratrol attenuates Hep3B (top), MHCC97-L (middle) and MHCC97-H (bottom) cells anchorage-independent cell growth. Soft agar assays were performed as described in Materials and methods. The asterisks indicate a significant $(" \mathrm{p}<0.05$, $\left.{ }^{* *} \mathrm{p}<0.01,{ }^{* * *} \mathrm{p}<0.001\right)$ decrease in cell viability or colony formation by resveratrol-treated cells.

protocol. Briefly, the slide was baked at $60^{\circ} \mathrm{C}$ for $2 \mathrm{~h}$, deparaffinized, and rehydrated. To expose the antigens, the slide was unmasked by submersion into boiling sodium citrate buffer (10 mM, pH 6.0) for $10 \mathrm{~min}$, and then treated with $3 \% \mathrm{H}_{2} \mathrm{O}_{2}$ for 10 min. The slide was blocked with $50 \%$ goat serum albumin in 1X PBS in a humidified chamber for $1 \mathrm{~h}$ at room temperature and then with a first antibody (1:100 dilution in 50\% goat serum with PBS) at $4^{\circ} \mathrm{C}$ in a humidified chamber overnight. The slide was washed and hybridized with the secondary antibody from Vector Laboratories (anti-rabbit 1:200) for $1 \mathrm{~h}$ at room temperature. Slides were stained using the Vectastain Elite ABC kit.

Statistical analysis. Standard statistical methods were performed using Statistics Package for Social Science (SPSS) software (version 13.0; SPSS, Chicago, IL, USA). All data are presented as mean values \pm SD as indicated and analyzed using the Student's t-test or ANOVA. A p-value $<0.05$ was considered statistically significant.

\section{Results}

Resveratrol inhibits HCC cell growth in vitro. First, we investigated the effects of resveratrol on cell viability in Hep3B, MHCC97-L and MHCC97-H cells. MTS data showed that resveratrol significantly decreased anchorage-dependent growth (Fig. 1A) of Hep3B, MHCC97-L and MHCC97-H cells in a dose-dependent manner. Moreover, treatment with resveratrol markedly inhibited anchorage-independent cell growth in all test HCC cells (Fig. 1B), and treated with $60 \mu \mathrm{M}$ resveratrol almost blocked the colony formation in soft agar. These results indicated that resveratrol inhibits HCC cell growth in vitro.

Resveratrol downregulates the c-Met signaling pathway. Previous studies have demonstrated that the c-Met signaling pathway is dysregulated in human HCC. Hyperactivation of c-Met, or its downstream target kinases Akt and ERK1/2 are always involved in HCC tumorigenic regulation. Herein, we 

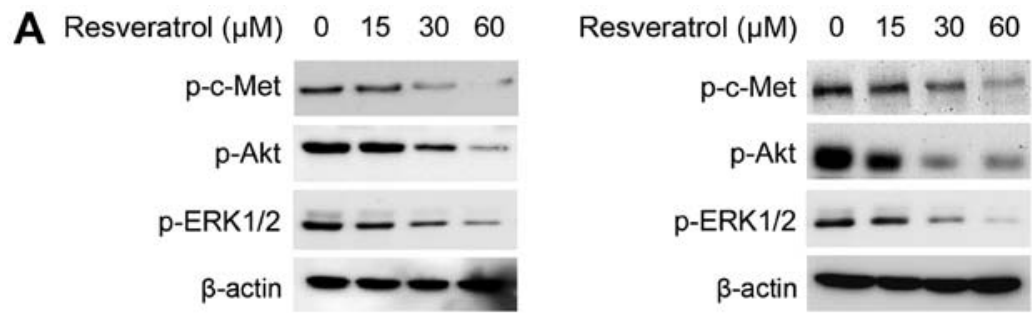

B
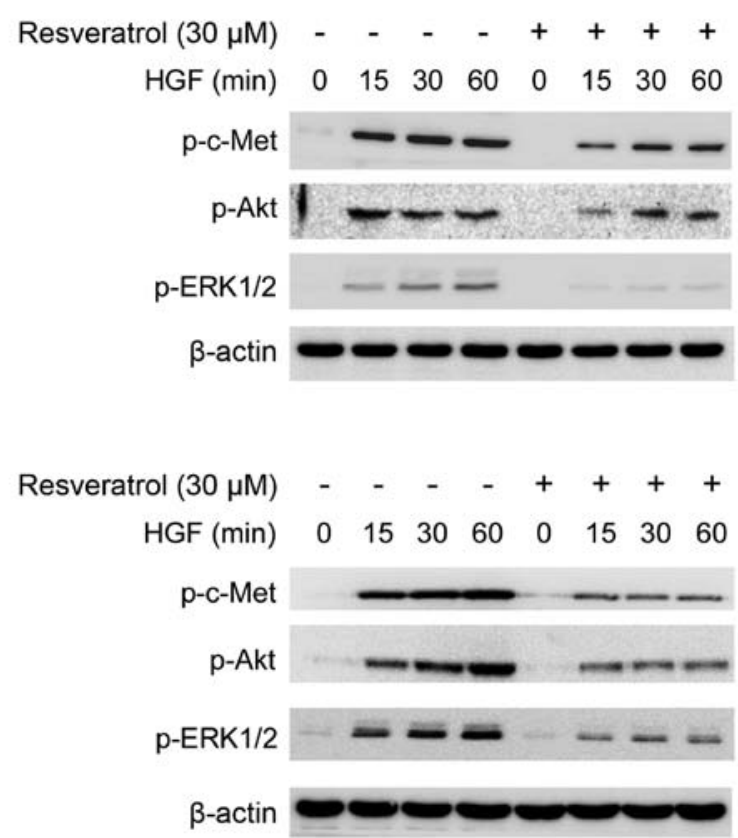

Figure 2. Resveratrol suppresses c-Met signaling pathway in HCC cells. (A) Resveratrol decreases the phosphorylation of c-Met signaling pathway. Hep3B (left) and MHCC97-H (right) cells were treated or not treated with resveratrol as indicated, cell lysates were harvested and protein levels were determined by western blot analysis. (B) Resveratrol affects HGF-induced c-Met signaling activation. Hep3B (top) and MHCC97-H (bottom) cells were starved in serum-free medium for $24 \mathrm{~h}$, after pre-treated with/without $30 \mu \mathrm{M}$ resveratrol for $2 \mathrm{~h}$, the cells were stimulated with HGF (10 ng/ml) for various times, cell lysates were harvested and protein levels were determined by western blot analysis.

A

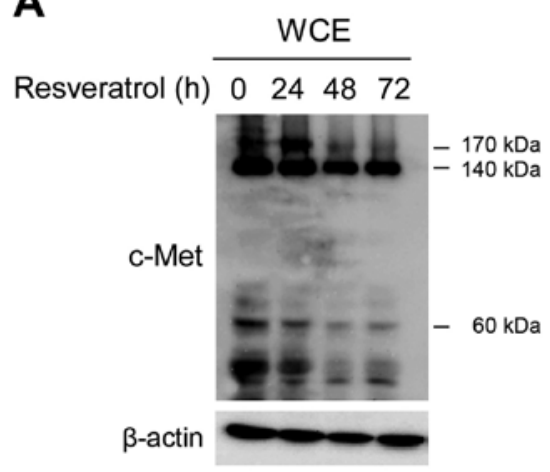

B

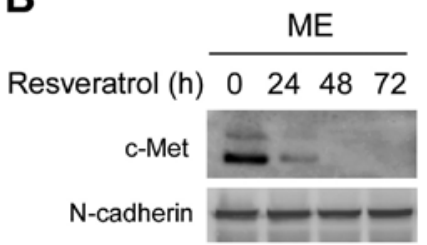

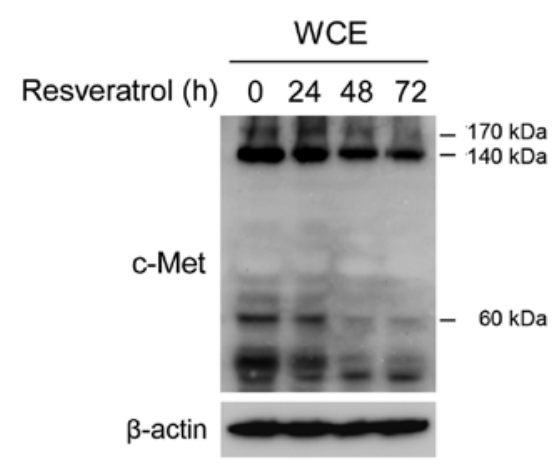

ME

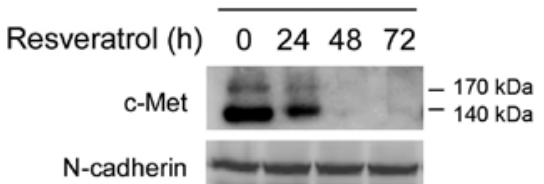

Figure 3. Resveratrol downregulates c-Met expression in HCC cells. (A) Resveratrol inhibits c-Met expression in Hep3B and MHCC97-H cells. Hep3B (left) and MHCC97-H (right) cells were treated with $60 \mu \mathrm{M}$ resveratrol for various time points, the whole cell lysates were extracted and protein levels were determined by western blot analysis. (B) Resveratrol decreases the expression of membrane-associated c-Met. Hep3B (left) and MHCC97-H (right) cells were treated with $60 \mu \mathrm{M}$ resveratrol for various time points, the membrane fractions were isolated and analyzed by western blotting using the indicated antibodies. 
A
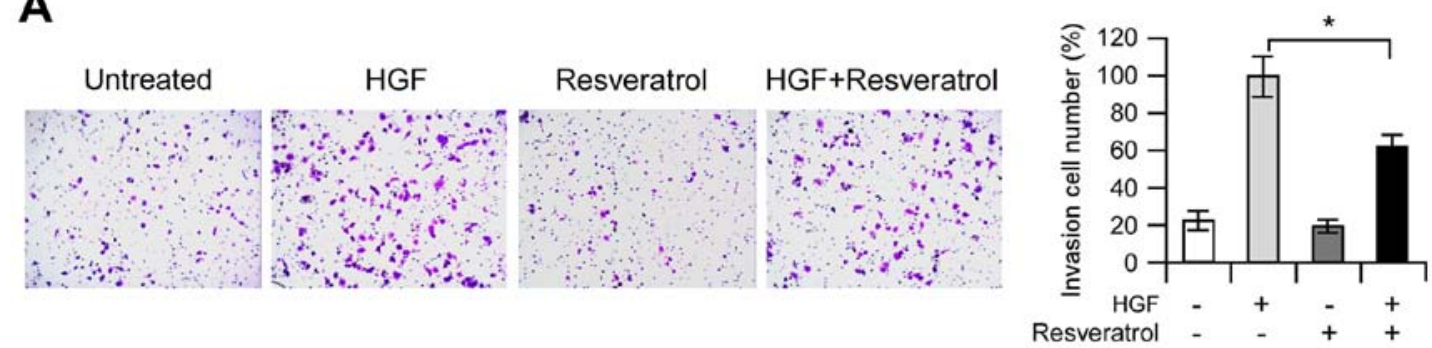

B
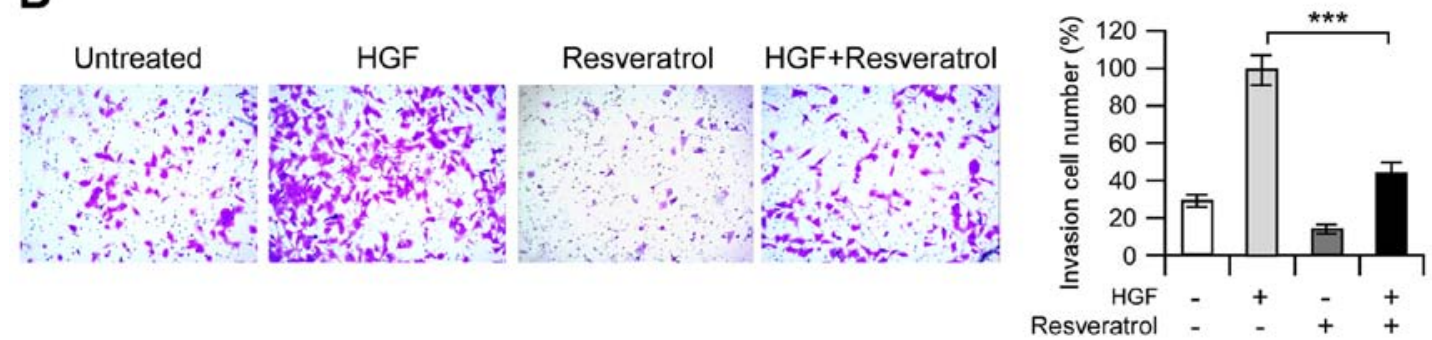

Figure 4. Resveratrol attenuates HGF-induced invasion in vitro. Hep3B (A) and MHCC97-H (B) cells were treated with resveratrol as described in Materials and methods. The cells adherent on lower surface of the filters were fixed and stained. Three representative fields were photographed under a light microscope and counted in triplicate to obtain invasion indices. The asterisks indicate a significant $\left({ }^{*} \mathrm{p}<0.05,{ }^{* * * *} \mathrm{p}<0.001\right)$ decrease of the invaded cells in resveratrol treated group.

found that resveratrol markedly impaired the phosphorylation of c-Met, Akt and ERK1/2 in a dose-dependent manner in Hep3B (Fig. 2A, left) and MHCC97-H cells (Fig. 2A, right). Moreover, we found that resveratrol treatment strikingly inhibited HGF-induced c-Met and downstream target kinases Akt and ERK1/2 activation in Hep3B (Fig. 2B, top) and MHCC97-H cells (Fig. 2B, bottom). Our data implied that resveratrol mediated growth inhibition may partly depend on the downregulation of c-Met signaling pathway.

Resveratrol suppresses the c-Met protein levels in HCC cells. Our data demonstrated that the c-Met signaling pathway was inhibited after short-term resveratrol treatment, which indicated that resveratrol could dose-dependently suppress the tyrosine kinase activity of c-Met. However, no published data have demonstrated that resveratrol directly regulates c-Met expression in human HCC. As shown in Fig. 3, longterm exposure $(24,48$ or $72 \mathrm{~h})$ to resveratrol decreased the protein levels of c-Met in a dose-dependent manner in Hep3B (Fig. 3A, left) and MHCC97-H cells (Fig. 3A, right). We further performed sub-cellular separation to investigate the effect of resveratrol on c-Met sub-cellular expression. As shown in Fig. 3B, membrane-associated c-Met was remarkably decreased after resveratrol treatment for $24 \mathrm{~h}$ in both Hep3B (Fig. 3B, left) and MHCC97-H cells (Fig. 3B, right), and at the time points of 48 and $72 \mathrm{~h}$, resveratrol almost blocked the membrane expression of c-Met. These results suggested that after the long-term treatment of resveratrol, the expression of c-Met on cell membrane was suppressed.

Resveratrol suppresses HGF-induced invasion in vitro. The HGF-c-Met signaling pathway has a crucial role in cancer cell invasion and metastasis. Based on previous data, to explore the potential anti-invasive effects of resveratrol, invasion assays were performed on Hep3B and MHCC97-H cells.
As shown in Fig. 3A, HGF substantially induced Hep3B cell's invasion, and resveratrol markedly inhibited the HGF-induced invasive capacity of malignant Hep3B cells (Fig. 4A). We also found that the MHCC97-H cells possessed much higher invasion capacity than Hep3B cell, even without HGF treatment. As expected, resveratrol showed very similar inhibitory effect against the invasion capacity of MHCC97-H cell (Fig. 4B). These results suggested that resveratrol might be an effective inhibitor for HGF-induced invasion of Hep3B and MHCC97-H cells.

Knockdown of c-Met decreases the sensitivity of MHCC97-H cells to resveratrol. We then examined whether knocking down c-Met expression influences the sensitivity of MHCC97-H cells to resveratrol. The efficiency of short hairpin RNA (shRNA) knockdown was examined, and the expression of C-Met was obviously decreased after shRNA transfection (Fig. 5A). Moreover, the growth of cells in soft agar also decreased after transfection compared with the GFP group (Fig. 5B, left). Resveratrol (30 $\mu \mathrm{M})$ inhibited anchorage-independent growth of MHCC97-H cells transfected with GFP shRNA by $\sim 80 \%$. In contrast, the inhibition was $<50 \%$ in MHCC97-H cells transfected with c-Met shRNA, indicating that MHCC97-H cells transfected with c-Met shRNAs were more resistant to resveratrol treatment (Fig. 5B, right). These results suggested that c-Met plays an important role in the sensitivity of MHCC97-H cells to the anti-proliferative effects of resveratrol.

Resveratrol inhibits tumor growth and c-Met phosphorylation in a xenograft mouse model. We examined the effects of resveratrol on tumor growth and c-Met activation using a human HCC xenograft. MHCC97-H cells were injected (s.c.) into the right flank of six-week-old female athymic nude mice. Data showed that resveratrol markedly reduced tumor size 
A

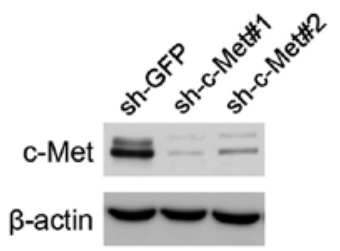

B
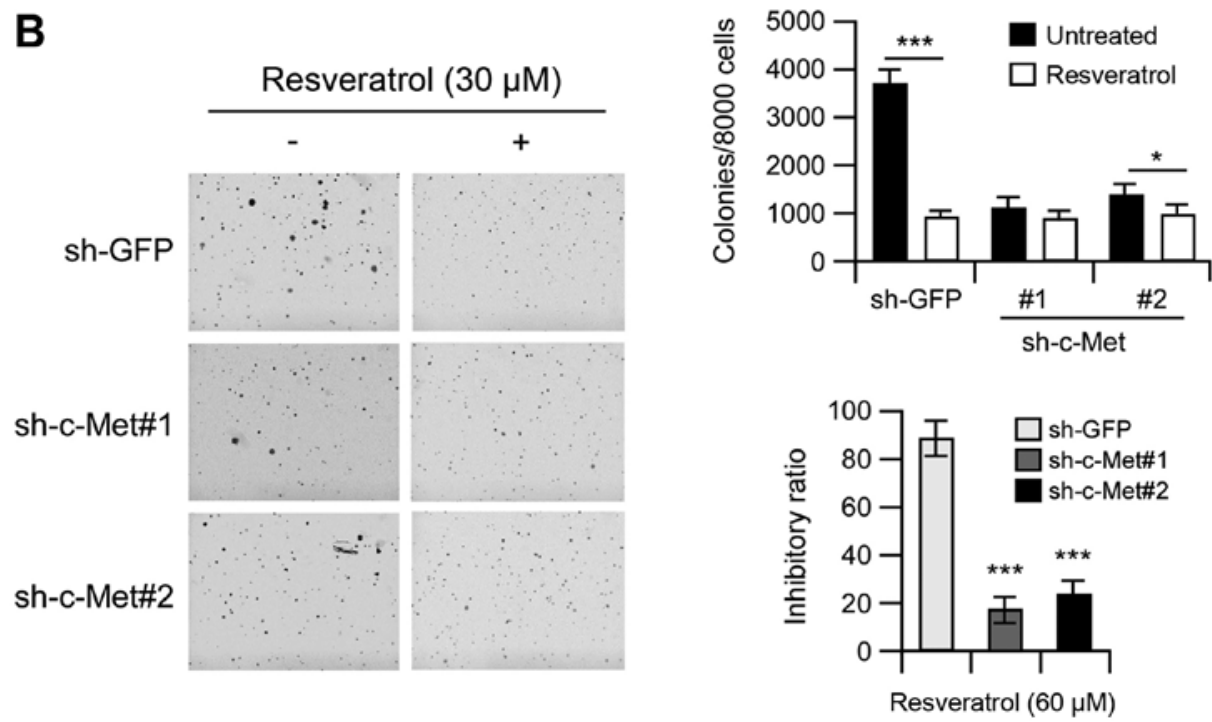

Figure 5. Knockdown of c-Met in MHCC97-H cells decreases the sensitivity to resveratrol. (A) Efficiency of c-Met shRNA in MHCC97-H cells. (B) anchorageindependent growth of MHCC97-H cells transfected with GFP shRNA or c-Met shRNA, treated or not treated with resveratrol. The asterisks indicate a significant $\left(" \mathrm{p}<0.05,{ }^{* * *} \mathrm{p}<0.001\right)$ decrease of the sensitivity in c-Met knockdown MHCC97-H cells.

in MHCC97-H xenograft model (Fig. 6A-C). Weights of all animals increased normally after treatment with resveratrol or vehicle control (Fig. 6D). Additionally, immunohistochemical analysis of resveratrol treated MHCC97-H xenograft tumors was conducted to evaluate the expression level of p-c-Met and $\mathrm{Ki}-67$. Our results showed that Ki-67 and p-c-Met were significantly suppressed in the resveratrol treated group compared with the vehicle treated group (Fig. 6E). These results clearly indicated that resveratrol inhibits tumor cell proliferation in vivo. In addition, these findings showed that resveratrol significantly inhibited tumor cell c-Met activation.

\section{Discussion}

Previous studies have demonstrated that resveratrol displayed potent antitumor effect in various types of tumors, including lung, breast, prostate and colon cancer $(23,26)$. Herein, we found that resveratrol inhibited hepatocellular carcinoma cell anchorage-dependent and -independent growth, as well as HGF-induced c-Met signaling pathway activation and tumor cell invasion. Importantly, for the first time, we also uncovered that exposure to resveratrol directly downregulated the membrane expression of c-Met. Knockdown of c-Met decreased the sensitivity of $\mathrm{HCC}$ cells to resveratrol treatment. Our in vivo data further demonstrated that resveratrol suppressed the HCC tumor growth in a xenograft mouse model.

Hepatocellular carcinoma, which accounts for $70-85 \%$ of the cases, is the most common primary liver cancer (27). Despite improvements in local therapies, including surgical resection, liver transplantation, and transarterial embolization, the prognosis remains poor for the majority of patients who develop recurrence, metastasis or present with advanced disease (28). HCC is a complex and heterogeneous tumor with several genomic alterations, the dysregulation of several signaling cascades such as PI3K/Akt, Ras/ERK, EGF/EGFR and $\mathrm{HGF} / \mathrm{c}-$ Met signaling pathways are involved in the initiation and progression of this kind of disease (29-32). Especially, ongoing efforts to study hepato-carcinogenesis have identified an important role for c-Met signaling in the promotion of tumor growth, angiogenesis, and metastasis. The transcription of c-Met is increased in 30-100\% of tumors compared with surrounding liver tissue. Similarly, c-Met is overexpressed at the protein level in $25-100 \%$ of HCCs compared with normal liver $(4,33)$. Importantly, recent studies suggest that c-Met overexpression is significantly associated with clinicopathological features of HCC, such as tumor grade, vascular invasion or thrombosis, tumor recurrence, metastases, and worse prognosis with impaired 5-year survival (34-36). This evidence suggests a potential tumor-promoting role of c-Met and the HGF-c-Met signaling pathway in HCC.

In the canonical HGF/c-Met signaling pathway, the activation of c-Met and downstream kinases dependent on the binding of HGF on c-Met receptor leads to c-Met homodimerization, autophosphorylation, and signal transduction. Moreover, c-Met binds with EGF receptor (EGFR) and some other receptor tyrosine kinases on cell membrane to activate downstream kinases, even in the absence of $\operatorname{HGF}(4,10,37)$. Regardless of the mode of activation, c-Met dimerization on cell membrane and autophosphorylation seem to be necessary for activation of downstream kinases in human malignant 
A

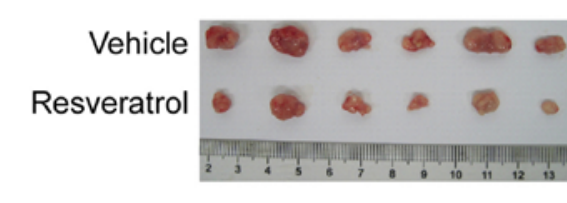

C

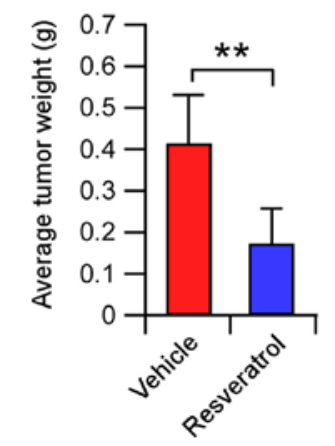

E

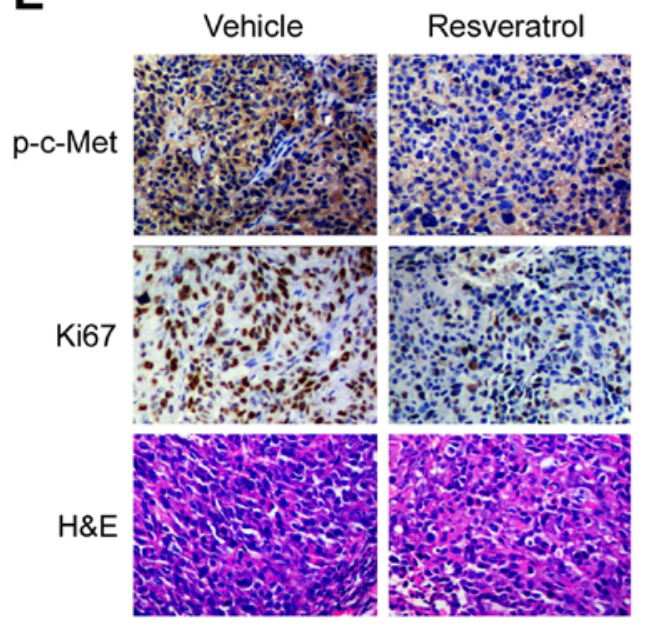

B

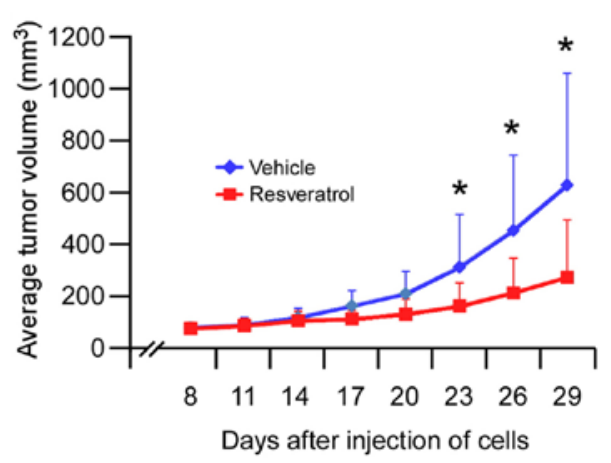

D

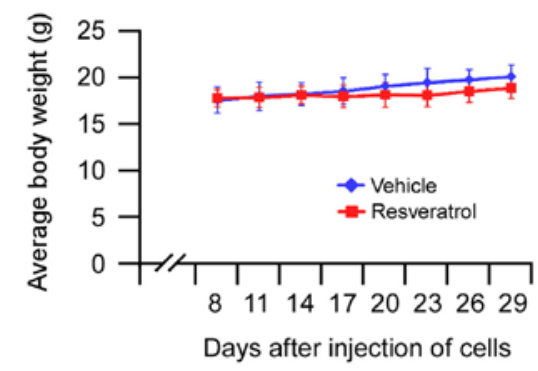

Figure 6. Resveratrol inhibits tumor growth in MHCC97-H xenograft mouse models. (A) At the treatment end point, mice were sacrificed and tumors were removed, weighed and photographed. (B) MHCC97-H cells were subcutaneously injected into the flank of mice. Tumor volumes were measured twice a week. (C) The tumor weight of mice from the vehicle group and resveratrol-treated group was measured. (D) During the treatment period, the body weight of mice was measured twice a week to determine the effect of resveratrol. (E) Immunohistochemical staining examination of Ki67 and p-c-Met in tumor sections from vehicle group mice or resveratrol-treated group mice. All panels are of the same magnification. Data are shown as mean values $\pm \mathrm{SD}$. $\left(\mathrm{p}<0.05,{ }^{* *} \mathrm{p}<0.01\right.$ versus vehicle-treated group).

tumors. In the present study, we firstly demonstrated that resveratrol not only decreased HGF-induced c-Met signaling activation, but also downregulated c-Met protein on cell membrane directly. Given resveratrol is a kind of flavonoid which is abundantly distributed in fruits and vegetables, our results suggested that a daily diet of high intake of resveratrol would be beneficial for HCC patients, especially for those c-Met-positive, or the tumors which are addicted to c-Met signaling pathways.

Our studies also found the antitumor effect of resveratrol was correlated with its effect on cancer cell motility and invasiveness. It is well known that the invasion and metastasis are the cause of $>90 \%$ of human cancer deaths, and c-Met plays a pivotal role in the process of tumor invasion and metastasis (9,38-40). c-Met signaling pathway regulates epithelial-mesenchymal transition in cancer cells, overexpression or hyperactivation of c-Met were involved in regulation of metastasis in multiple different types of human cancer, including lung (41), liver (42) and breast (14) cancer. Additionally, transgenic mice overexpressing HGF exhibited increased angiogenesis and VEGF transcription in chemically-induced hepatic adenomas and HCC (43). Ogunwobi et al reported that epigenetic upregulation of HGF and c-Met drives metastasis in hepatocellular carcinoma (44), which indicated that the promotion of metastasis in HCC, at least partly, depend on the HGF-c-Met pathway.

Our in vitro data also demonstrated that HGF-induced invasion of HCC cells was significantly decreased after resveratrol treatment. Our results implied that combination of resveratrol with other anti-angiogenetic agents may have synergistic effect on invasion or metastasis suppression.

Crosstalk between c-Met and other RTKs on cell membrane has also been studied in great depth because of its potential importance in the development of resistance 
to cancer therapeutics (17). For instance, the amplification of c-Met is one of the main reasons for lung cancer cells gaining acquired resistance to gefitinib treatment (45). Importantly, c-Met has also been shown by multiple studies to interact directly with the epidermal growth factor receptor (EGFR) on membrane, allowing activation of c-Met after stimulation with the EGFR ligands EGF or transforming growth factor (TGF- $\alpha$ ) (46). Additionally, treatment with EGF in cells expressing both c-Met and EGFR directly induced phosphorylation of c-Met (47). In cancer cells, c-Met interacts with other ERBB family members, such as ERBB2 and ERBB3, causing transactivation of both receptors (48). Recently, the ligand-independent activation of c-Met through the interaction with IGF-1R, was also demonstrated in prostate cancer in an IGF-1/IGF-1R signaling pathway-dependent manner (49). Unexpectedly, our results uncovered for the first time that exposure to resveratrol directly downregulated the expression of c-Met on the cell membrane. The data suggested that resveratrol not only inhibited HGF-induced c-Met receptor tyrosine kinase activity but also directly decreased the expression of c-Met on the cell membrane.

Overall, for the first time, the antitumor activity of resveratrol against HCC via suppression of c-Met signaling pathway was investigated both in vitro and in vivo.

Similar to other natural compounds, such as EGCG (50) and quercetin (51), resveratrol has also been demonstrated as a multiple kinase inhibitor in human cancers. Herein, the downregulation of HGF-c-Met signaling might not be specific, other signaling pathways and mechanisms also involved in resveratrol-mediated HCC suppression will need further study in the nearly future. Despite the large number of pre-clinical studies dealing with different aspects of the biological effects of resveratrol, its translation to clinics for cancer therapies is far from reality due to a variety of challenges. Different from the mechanism reported by previous studies, we demonstrated that the c-Met signaling pathway was one of the underlying mechanisms for resveratrol to exert its tumor fighting effect. Our study implied that c-Met is a potent antitumor target to be applied to prevention and clinical treatment of human hepatocellular carcinoma.

\section{Acknowledgements}

This work was supported by National Natural Science Foundation of China (no. 81371690), Research Project of Health and Family Planning Commission of Hunan Province (B2016125), and Science and Technology Program Project Funds of Changsha (K1403054-31, K15ZD052-33).

\section{References}

1. Ferlay J, Shin HR, Bray F, Forman D, Mathers C and Parkin DM: Estimates of worldwide burden of cancer in 2008: GLOBOCAN 2008. Int J Cancer 127: 2893-2917, 2010.

2. Perz JF, Armstrong GL, Farrington LA, Hutin YJ and Bell BP: The contributions of hepatitis $B$ virus and hepatitis $C$ virus infections to cirrhosis and primary liver cancer worldwide. J Hepatol 45: 529-538, 2006.

3. Seton-Rogers S: Hepatocellular carcinoma: Gender differences. Nat Rev Cancer 14: 578-579, 2014.

4. Goyal L, Muzumdar MD and Zhu AX: Targeting the HGF/c-MET pathway in hepatocellular carcinoma. Clin Cancer Res 19: 2310-2318, 2013
5. Llovet JM, Villanueva A, Lachenmayer A and Finn RS: Advances in targeted therapies for hepatocellular carcinoma in the genomic era. Nat Rev Clin Oncol 12: 408-424, 2015.

6. Forner A, Gilabert M, Bruix J and Raoul JL: Treatment of intermediate-stage hepatocellular carcinoma. Nat Rev Clin Oncol 11: 525-535, 2014.

7. Liu X, Wang Q, Yang G, Marando C, Koblish HK, Hall LM, Fridman JS, Behshad E, Wynn R, Li Y, et al: A novel kinase inhibitor, INCB28060, blocks c-MET-dependent signaling, neoplastic activities, and cross-talk with EGFR and HER-3. Clin Cancer Res 17: 7127-7138, 2011.

8. Peters S and Adjei AA: MET: A promising anticancer therapeutic target. Nat Rev Clin Oncol 9: 314-326, 2012.

9. Gherardi E, Birchmeier W, Birchmeier C and Vande Woude G: Targeting MET in cancer: Rationale and progress. Nat Rev Cancer 12: 89-103, 2012.

10. You H, Ding W, Dang H, Jiang Y and Rountree CB: c-Met represents a potential therapeutic target for personalized treatment in hepatocellular carcinoma. Hepatology 54: 879-889, 2011.

11. Kim IH, Lee IH, Lee JE, Hong SH, Kim TJ, Lee KY, Kim YK, Kim SJ, Sung SW, Park JK, et al: Prognostic impact of multiple clinicopathologic risk factors and c-Met overexpression in patients who have undergone resection of stage IB non-small-cell lung cancer. Clin Lung Cancer 17: e31-e43, 2016.

12. Burggraaf J, Kamerling IM, Gordon PB, Schrier L, de Kam ML, Kales AJ, Bendiksen R, Indrevoll B, Bjerke RM, Moestue SA, et al: Detection of colorectal polyps in humans using an intravenously administered fluorescent peptide targeted against c-Met. Nat Med 21: 955-961, 2015.

13. Otte A, Rauprich F, von der Ohe J, Yang Y, Kommoss F, Feuerhake F, Hillemanns P and Hass R: c-Met inhibitors attenuate tumor growth of small cell hypercalcemic ovarian carcinoma (SCCOHT) populations. Oncotarget 6: 31640-31658, 2015.

14. Xing F, Liu Y, Sharma S, Wu K, Chan MD, Lo HW, Carpenter RL, Metheny-Barlow LJ, Zhou X, Qasem SA, et al: Activation of the c-Met pathway mobilizes an inflammatory network in the brain microenvironment to promote brain metastasis of breast cancer. Cancer Res 76: 4970-4980, 2016.

15. Marano L, Chiari R, Fabozzi A, De Vita F, Boccardi V, Roviello G, Petrioli R, Marrelli D, Roviello F and Patriti A: c-Met targeting in advanced gastric cancer: An open challenge. Cancer Lett 365: 30-36, 2015.

16. Zhao Z, Zhu X, Cui K, Mancuso J, Federley R, Fischer K, Teng GJ, Mittal V, Gao D, Zhao H, et al: In vivo visualization and characterization of epithelial-mesenchymal transition in breast tumors. Cancer Res 76: 2094-2104, 2016.

17. Organ SL and Tsao MS: An overview of the c-MET signaling pathway. Ther Adv Med Oncol 3 (Suppl): S7-S19, 2011.

18. Medina-Aguilar R, Pérez-Plasencia C, Marchat LA, Gariglio P, García Mena J, Rodríguez Cuevas S, Ruíz-García E, Astudillo-de la Vega H, Hernández Juárez J, Flores-Pérez A, et al: Methylation landscape of human breast cancer cells in response to dietary compound resveratrol.PLoS One 11: e0157866, 2016.

19. Buhrmann C, Shayan P, Popper B, Goel A and Shakibaei M: Sirt1 is required for resveratrol-mediated chemopreventive effects in colorectal cancer cells. Nutrients 8: 145, 2016.

20. Gwak H, Kim S, Dhanasekaran DN and Song YS: Resveratrol triggers ER stress-mediated apoptosis by disrupting N-linked glycosylation of proteins in ovarian cancer cells. Cancer Lett 371: 347-353, 2016.

21. Sahin E, Baycu C, Koparal AT, Burukoglu Donmez D and Bektur E: Resveratrol reduces IL-6 and VEGF secretion from co-cultured A549 lung cancer cells and adipose-derived mesenchymal stem cells. Tumour Biol 37: 7573-7582, 2016.

22. Taniguchi T, Iizumi Y, Watanabe M, Masuda M, Morita M, Aono Y, Toriyama S, Oishi M, Goi W and Sakai T: Resveratrol directly targets DDX5 resulting in suppression of the mTORC1 pathway in prostate cancer. Cell Death Dis 7: e2211, 2016.

23. Singh CK, Ndiaye MA and Ahmad N: Resveratrol and cancer: Challenges for clinical translation. Biochim Biophys Acta 1852: 1178-1185, 2015.

24. Yu X, Deng Q, Li W, Xiao L, Luo X, Liu X, Yang L, Peng S, Ding Z, Feng T, et al: Neoalbaconol induces cell death through necroptosis by regulating RIPK-dependent autocrine $\mathrm{TNF} \alpha$ and ROS production. Oncotarget 6: 1995-2008, 2015.

25. Li W, Peng C, Lee MH, Lim D, Zhu F, Fu Y, Yang G, Sheng Y, Xiao L, Dong X, et al: TRAF4 is a critical molecule for Akt activation in lung cancer. Cancer Res 73: 6938-6950, 2013. 
26. Kulkarni SS and Cantó C: The molecular targets of resveratrol Biochim Biophys Acta 1852: 1114-1123, 2015.

27. Davis GL, Dempster J, Meler JD, Orr DW, Walberg MW, Brown B, Berger BD, O'Connor JK and Goldstein RM: Hepatocellular carcinoma: Management of an increasingly common problem. Proc (Bayl Univ Med Cent) 21: 266-280, 2008.

28. Llovet JM and Bruix J: Molecular targeted therapies in hepatocellular carcinoma. Hepatology 48: 1312-1327, 2008.

29. Cui SX, Shi WN, Song ZY, Wang SQ, Yu XF, Gao ZH and Qu XJ: Des-gamma-carboxy prothrombin antagonizes the effects of Sorafenib on human hepatocellular carcinoma through activation of the Raf/MEK/ERK and PI3K/Akt/mTOR signaling pathways. Oncotarget 7: 36767-36782, 2016.

30. Li K, Shen B, Cheng X, Ma D, Jing X, Liu X, Yang W, Peng C and Qiu W: Phenotypic and signaling consequences of a nove aberrantly spliced transcript FGF receptor-3 in hepatocellular carcinoma. Cancer Res 76: 4205-4215, 2016.

31. Lanaya H, Natarajan A, Komposch K, Li L, Amberg N, Chen L Wculek SK, Hammer M, Zenz R, Peck-Radosavljevic M, et al: EGFR has a tumour-promoting role in liver macrophages during hepatocellular carcinoma formation. Nat Cell Biol 16: 972-981, 971-977, 2014.

32. Lau EY, Lo J, Cheng BY, Ma MK, Lee JM, Ng JK, Chai S, Lin $\mathrm{CH}$, Tsang SY, Ma S, et al: Cancer-associated fibroblasts regulate tumor-initiating cell plasticity in hepatocellular carcinoma through c-Met/FRA1/HEY1 signaling. Cell Rep 15: $1175-1189,2016$

33. Wu F, Wu L, Zheng S, Ding W, Teng L, Wang Z, Ma Z and Zhao W: The clinical value of hepatocyte growth factor and its receptor - c-met for liver cancer patients with hepatectomy. Dig Liver Dis 38: 490-497, 2006.

34. Suzuki K, Hayashi N, Yamada Y, Yoshihara H, Miyamoto Y, Ito Y, Ito T, Katayama K, Sasaki Y, Ito A, et al: Expression of the c-met protooncogene in human hepatocellular carcinoma. Hepatology 20: 1231-1236, 1994

35. Choi KJ, Baik IH, Ye SK and Lee YH: Molecular targeted therapy for hepatocellular carcinoma: Present status and future directions. Biol Pharm Bull 38: 986-991, 2015.

36. Torrecilla S and Llovet JM: New molecular therapies for hepatocellular carcinoma. Clin Res Hepatol Gastroenterol 39 (Suppl 1): S80-S85, 2015

37. Suzuki M, Shiraha H, Fujikawa T, Takaoka N, Ueda N, Nakanishi Y, Koike K, Takaki A and Shiratori Y: Des-gammacarboxy prothrombin is a potential autologous growth factor for hepatocellular carcinoma. J Biol Chem 280: 6409-6415, 2005.

38. Spano D, Heck C, De Antonellis P, Christofori G and Zollo M Molecular networks that regulate cancer metastasis. Semin Cancer Biol 22: 234-249, 2012.

39. Mehlen P and Puisieux A: Metastasis: A question of life or death. Nat Rev Cancer 6: 449-458, 2006
40. Feng Y, Thiagarajan PS and Ma PC: MET signaling: Novel targeted inhibition and its clinical development in lung cancer. J Thorac Oncol 7: 459-467, 2012.

41. Chen QY, Jiao DM, Wu YQ, Chen J, Wang J, Tang XL, Mou H, $\mathrm{Hu}$ HZ, Song J, Yan J, et al: MiR-206 inhibits HGF-induced epithelial-mesenchymal transition and angiogenesis in non-small cell lung cancer via c-Met /PI3k/Akt/mTOR pathway. Oncotarget 7: 18247-18261, 2016.

42. Wang Y, Zhan Z, Jiang X, Peng X, Shen Y, Chen F, Ji Y, Liu W, Shi Y, Duan W, et al: Simm530, a novel and highly selective c-Met inhibitor, blocks c-Met-stimulated signaling and neoplastic activities. Oncotarget 7: 38091-38104, 2016.

43. Horiguchi N, Takayama H, Toyoda M, Otsuka T, Fukusato T, Merlino G, Takagi H and Mori M: Hepatocyte growth factor promotes hepatocarcinogenesis through c-Met autocrine activation and enhanced angiogenesis in transgenic mice treated with diethylnitrosamine. Oncogene 21: 1791-1799, 2002.

44. Ogunwobi OO, Puszyk W, Dong HJ and Liu C: Epigenetic upregulation of HGF and c-Met drives metastasis in hepatocellular carcinoma. PLoS One 8: e63765, 2013.

45. Engelman JA, Zejnullahu K, Mitsudomi T, Song Y, Hyland C, Park JO, Lindeman N, Gale CM, Zhao X, Christensen J, et al: MET amplification leads to gefitinib resistance in lung cancer by activating ERBB3 signaling. Science 316: 1039-1043, 2007.

46. Jo M, Stolz DB, Esplen JE, Dorko K, Michalopoulos GK and Strom SC: Cross-talk between epidermal growth factor receptor and c-Met signal pathways in transformed cells. J Biol Chem 275: 8806-8811, 2000

47. Puri N and Salgia R: Synergism of EGFR and c-Met pathways, cross-talk and inhibition, in non-small cell lung cancer. J Carcinog 7: 9, 2008.

48. Bachleitner-Hofmann T, Sun MY, Chen CT, Tang L, Song L, Zeng Z, Shah M, Christensen JG, Rosen N, Solit DB, et al: HER kinase activation confers resistance to MET tyrosine kinase inhibition in MET oncogene-addicted gastric cancer cells. Mol Cancer Ther 7: 3499-3508, 2008

49. Varkaris A, Gaur S, Parikh NU, Song JH, Dayyani F, Jin JK, Logothetis CJ and Gallick GE: Ligand-independent activation of MET through IGF-1/IGF-1R signaling. Int J Cancer 133: 1536-1546, 2013

50. Peter B, Bosze S and Horvath R: Biophysical characteristics of proteins and living cells exposed to the green tea polyphenol epigallocatechin-3-gallate (EGCg): Review of recent advances from molecular mechanisms to nanomedicine and clinical trials. Eur Biophys J: Jun 16, 2016 (Epub ahead of print).

51. Khan F, Niaz K, Maqbool F, Ismail Hassan F, Abdollahi M, Nagulapalli Venkata KC, Nabavi SM and Bishayee A: Molecular targets underlying the anticancer effects of quercetin: An update. Nutrients 8: pii: E529, 2016. doi: 10.3390/nu8090529. 